\title{
Effects of Munari powder on physical and sensory-motor parameters: a preliminary report
}

\author{
Nejc Sarabon $(1,2)$ \\ (1) University of Primorska, Andrej Marusic Institute, Department of Health Study, Koper, \\ Slovenia; (2) S2P, Science to Practice, d.o.o., Laboratory for Motor Control and Motor \\ Behaviour, Ljubljana, Slovenia
}

\begin{abstract}
Munari powder is broadly used in physical medicine and rehabilitation to decrease pain and help normalize sensory-motor function. It operates as TPRV1 agonist and "stops" generation of action potentials in pain nerve fibers. This is a short report of a pilot study on 20 subjects. Every subject underwent four visits to our laboratory, where the Munari applications and related measurements of its effects took place. Each of the healthy adults received the following applications: (1) placebo, i.e. $0 \%$ cayenne pepper mixture, consisting only of water and kaolin, (2) weak, i.e. $2.5 \%$ cayenne pepper mixture, (3) medium, i.e. $5.0 \%$ cayenne pepper mixture, and (4) strong, i.e. 10\% cayenne pepper mixture. The assessments were carried out before the Munari powder patch application, right after the application, and 15 and 30 min after the termination of the 20-minute Munari powder patch application. We measured subjective cold/hot feeling on visual analogue scale, blood pressure, body temperature, skin light touch sensations, sense for two-point discrimination, and pain threshold to the mechanical stimulus.-Besides these tests, maximal voluntary force during isometric trunk extension and the sitting balance test were performed. The preliminary results indicate that the 5\% concentration of cayenne pepper mixture is the best choice because no additional effects were observed with the $10 \%$ concentration and the effects are higher than with $2.5 \%$ concentration. Whether this will be also thrue for the patients suffering pain ought to be determined.
\end{abstract}

Key Words: pain, therapy, herbal, cayenne pepper

Eur J Transl Myol - Basic Appl Myol 2015; 25 (3): 191-194

Munari powder is broadly used in physical medicine and rehabilitation to decrease pain and help normalize sensory-motor function. It operates as TPRV1 agonist and "stops" generation of action potentials in pain nerve fibers (Figure 1 and Figure 2).

\section{Materials and Methods}

What we did was a pilot study on 20 subjects. Every subject underwent four visits to our laboratory, where the Munari applications and related measurements of its effects took part. A mixture of Munari consists of kaolin, cayenne pepper and water. $^{1-3}$ The main substance, however, is cayenne pepper.

Each of the healthy adults subjects received the following applications: (1) placebo, i.e. 0\% cayenne pepper mixture, consisting only of water and kaolin, (2) weak, i.e. $2.5 \%$ cayenne pepper mixture, (3) medium, i.e. $5.0 \%$ cayenne pepper mixture, and (4) strong, i.e. $10 \%$ cayenne pepper mixture. The assessments were carried out before the Munari powder patch application, right after the application, and 15 and 30 min after the termination of the 20minute Munari powder patch application. The decision about the 20 min duration was done based on the standard clinical application routine. We measured subjective cold/hot feeling on visual analogue scale (VAS), blood pressure, body temperature using thermos camera and we also did a monofilament test for light touch skin sensory function. ${ }^{4}$ For the latter test, fine filaments of different (very low) stiffness are being used, pressed perpendicular against the skin, and the subject is asked to report when feeling the touch. During this test, we started with the finest of the five filaments and progressed to the thicker filaments, using three touches/repetitions with each of the monofilaments. The thinnest monofilament, at which the subject correctly reported sensing the touch (at least two out of three), was considered the outcome result. Another test we used to test the effects of Munari on skin sensory functions was the two-point discrimination test. During this test, two metal pins are being pressed against the skin and the subject is asked 


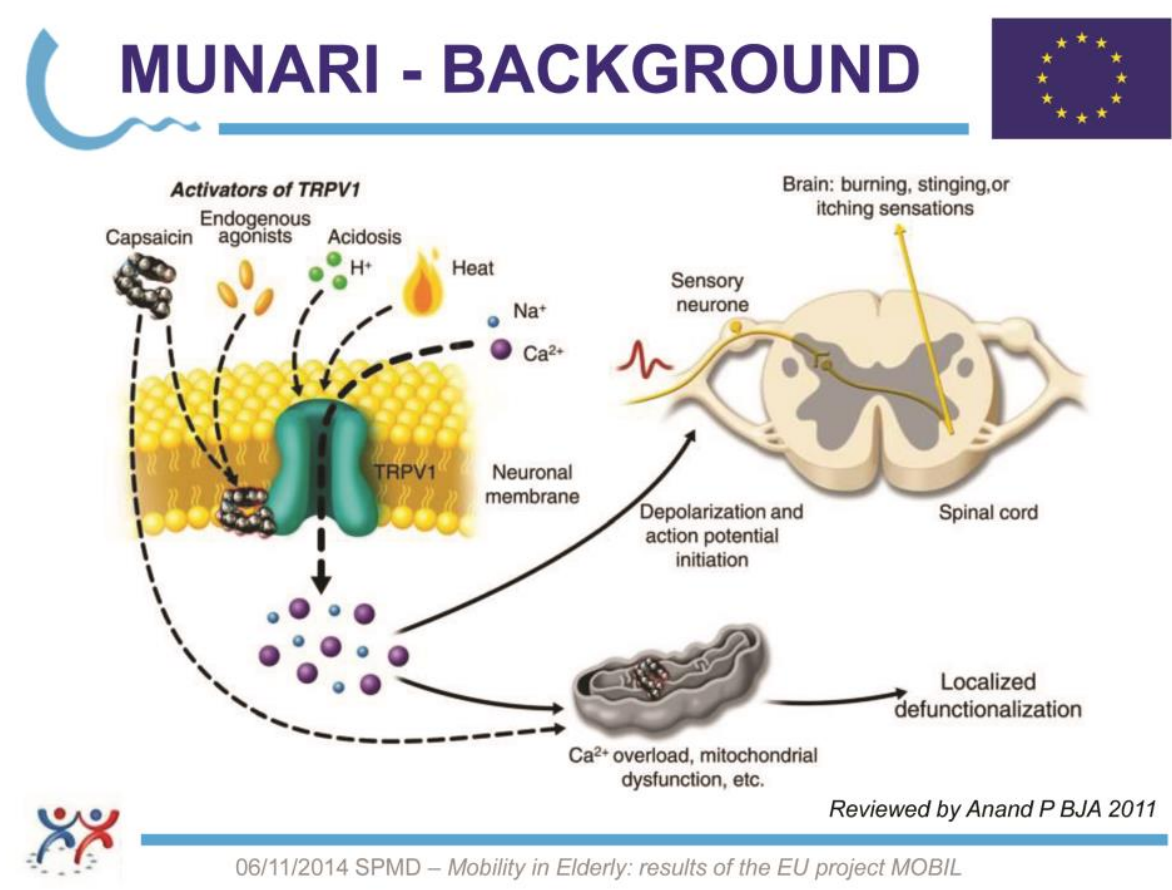

Fig 1. Action of capsaicin on the human sensory function.

to report if he/she felt a single or a double pin-touch. In order to exclude psychological moment and to increase validity of the test, we purposefully apply also singlepin touches during the testing routine - to be sure the subject is not guessing about the sensory experience. Letting the subjects know this procedure, resulted in a high level of attention, which is very much needed during this test. The inter-pin distance at which the subject felt two pins (at least two out of three repetitions) was noted as the result of the test. The next test was the mechanical algometry test. During this test, the $1 \mathrm{~cm}^{2}$ pin of the mechanical algometer was pressed perpendicular to the skin. The pressure was gradually increased until the subject reported pain.

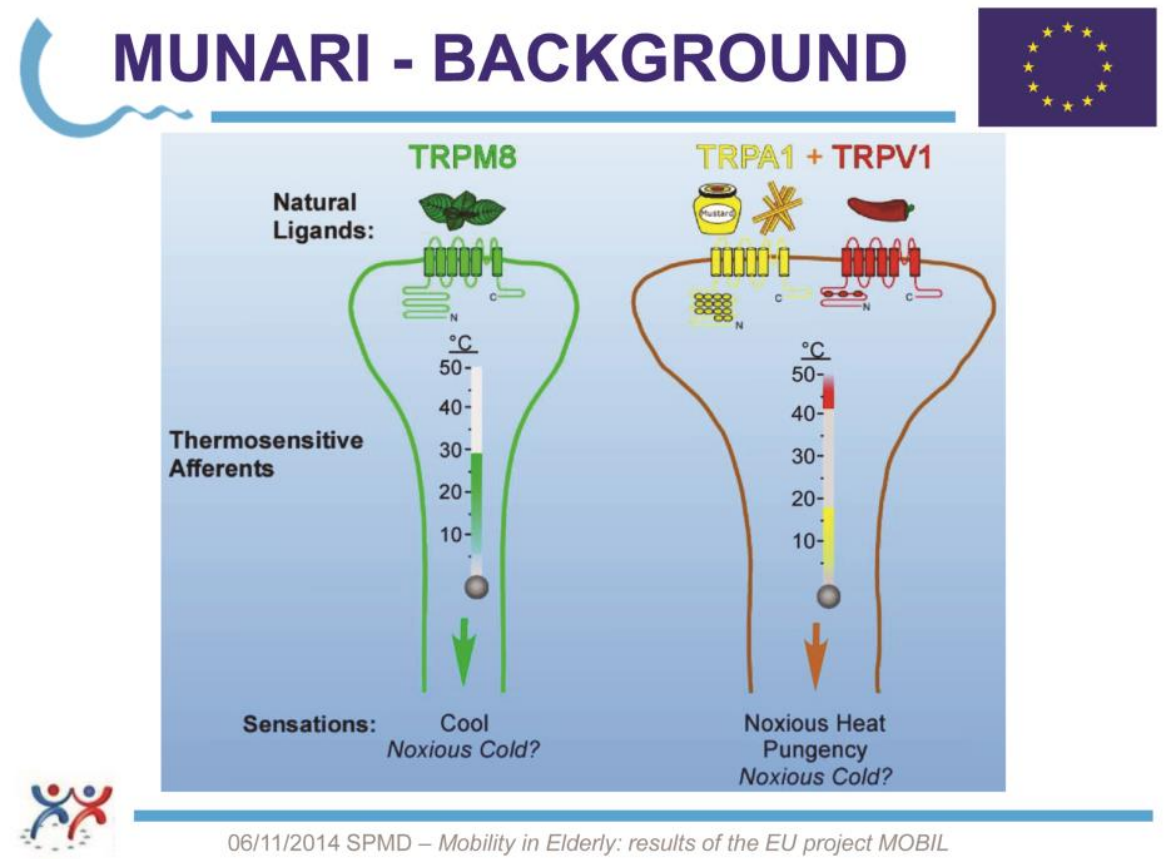

Fig 2. Thermal sensation modulatory action of different herbal applications. 


\section{Effects of Munari powder on physical and sensory-motor parameters}

Eur J Transl Myol - Basic Appl Myol 2015; 25 (3): 191-194

Another sensory test which we used was the trunk active repositioning test. The subject was asked to regain the position of the trunk flexion, to which he/she was guided in advance and asked to remember it. The three-repetition repositioning error was used as a result of this kinesthetic sense test. Besides these tests, we also carried out maximal voluntary force during isometric trunk extension and the sitting balance test (30 s sitting on a wobble board on the top of the force plate - quantification through centre-of-pressure parameters).

In order to learn the clinical procedure of Munari application, we, the researchers involved in this study, visited physical medicine and rehabilitation clinic in St. Pölten. Consistent with specific goals of our study, we received training on standard Munari application over the entire back region. Munari powder was heated up to 55 degrees Celsius and then applied over the entire back for $20 \mathrm{~min}$.

\section{Results and Discussion}

Here, we report some preliminary results. The skin temperature decreased in all concentrations (decrease of about $1.5^{\circ}$ Celsius). The effects in some of the measured parameters of sensory functions were stronger for $5 \%$ than for $2.5 \%$ concentration. For great majority of the parameters, no additional statistically significant effect was observed with the $10 \%$ concentration; compared to the $5 \%$ concentration. The values for heat sensation increased, but also went down $30 \mathrm{~min}$ after the application. The blood pressure did not changed. In the two-point discrimination test we saw an average increase from $55 \mathrm{~mm}$ to $61 \mathrm{~mm}$. The light touch skin sensation also decreased and we also saw a drop in sensitivity during pressure algometry. The balance and strength parameters were unchanged. These preliminary results indicate that the $5 \%$ concentration is the best because no additional effects can be indicated with the $10 \%$ concentration and the effects are higher than with $2.5 \%$ concentration. In lower back pain patients we think the outcome might be very different because here we are dealing with intact persons, who had no functional limitations. Also, the sensation can be different in chronic pain conditions. So, this is our next step - to proceed with research on Munari application effects in patients suffering from musculoskeletal pain.

We need to define our research aim and hypothesis good enough to be able to add new information.

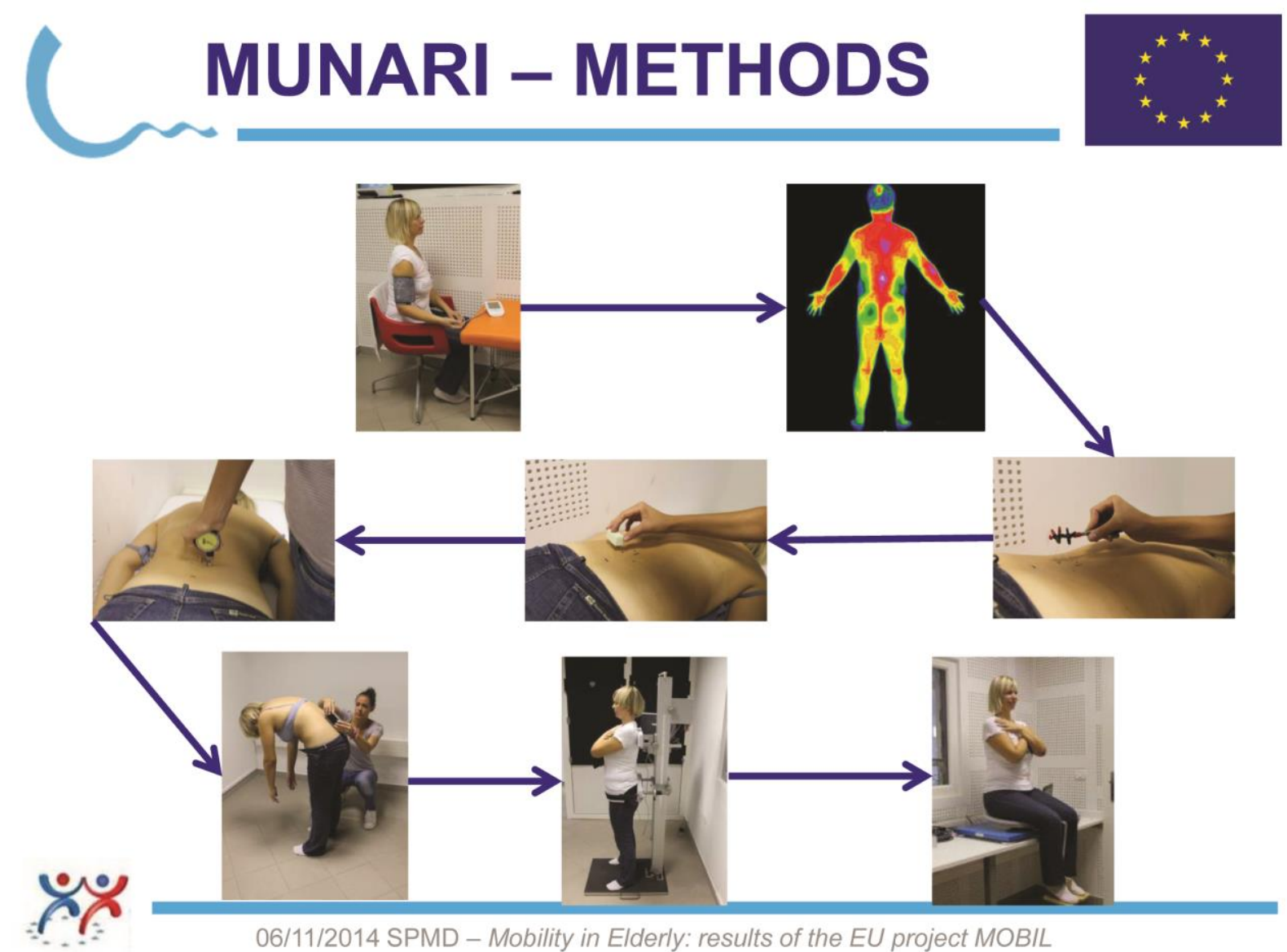

Fig 3. Schematic presentation of the measurements/application as used in this study. 


\section{Effects of Munari powder on physical and sensory-motor parameters}

Eur J Transl Myol - Basic Appl Myol 2015; 25 (3): 191-194

\section{Acknowledgements}

This paper is a transcription of the talk presented to the Mobility in Elderly Result Meeting, November 8 2014, Vienna, Austria. The Author thanks for invitation.

\section{Corresponding Author}

Nejc Sarabon

University of Primorska, Andrej Marusic Institute, Department of Health Study, Muzejski trg 2, SI-6000

Koper, Slovenia. E-mail: nejc.sarabon@s2p.si

\section{References}

1. Chrubasik C, Black A, Müller-Ladner U, Chrubasik S. Impact of herbal medicines on physical impairment. Phytomed 2008;15:536-9. doi:10.1016/j.phymed.2007.09.007.
2. Rubinstein SM, van Middelkoop M, Kuijpers T, et al. A systematic review on the effectiveness of complementary and alternative medicine for chronic non-specific low-back pain. Eur Spine J 2010;19:1213-28. doi:10.1007/s00586-010-13563.

3. Gagnier JJ, van Tulder MW, Berman B, Bombardier C. Herbal medicine for low back pain: a Cochrane review. Spine (Phila Pa 1976). 2007;32(1):82-92. doi:10.1097/01.brs.0000249525.70011.fe.

4. Descarreaux M, Blouin J-S, Teasdale N. Repositioning accuracy and movement parameters in low back pain subjects and healthy control subjects. Eur Spine J. 2005;14:185-91. doi:10.1007/s00586-004-0833-y.- 